Annals of Pure and Applied Mathematics

Vol. 14, No. 2, 2017, 337-345

Annals of

ISSN: 2279-087X (P), 2279-0888(online)

Published on 30 September 2017

www.researchmathsci.org

DOI: http://dx.doi.org/10.22457/apam.v14n2a17

Pure and Applied

Mathematics

\title{
On the Wiener Index of Some Total Graphs
}

\author{
Pravin Garg $^{1}$ and Shanu Goyal \\ ${ }^{1}$ Department of Mathematics, University of Rajasthan, Jaipur-302004 \\ Rajasthan, India. E-mail: garg.pravin@gmail.com \\ ${ }^{2}$ Department of Mathematics \& Statistics, Banasthali University, Banasthali-304022 \\ Rajasthan, India. E-mail: shanugoyalnewai@gmail.com \\ ${ }^{1}$ Corresponding author
}

Received 14 July 2017; accepted 1 August 2017

Abstract. The total graph $T(G)$ of a graph $G=(V, E)$ is that graph whose vertex set is $V \cup E$, and two vertices are adjacent if and only if they are adjacent or incident in $G$. For a fan graph $F_{1, n}$, the graph $F_{1, n} . F_{1, m}$ is obtained by identifying a vertex corresponding to $\bar{K}_{1}$ of $F_{1, n}$ to one vertex corresponding to $\bar{K}_{1}$ of $F_{1, m}$. In this note, we investigate the Wiener index of the total graphs of $F_{1, n} . F_{1, m}, K_{m, n}, F_{1, n}, F_{2, n}$ and $K_{2} \mathrm{~W} C_{n}$.

Keywords: Wiener index; total graph; fan graph

AMS Mathematics Subject Classification (2010): 05C12, 05 C76

\section{Introduction}

A fan graph $F_{m, n}$ is defined as the graph join $\bar{K}_{m}+P_{n}$, where $\bar{K}_{m}$ is the empty graph on $m$ nodes and $P_{n}$ is the path graph on $n$ vertices. The case $m=1$ corresponds to the usual fan graphs, while $m=2$ corresponds to the double fan graph. A bipartite graph is a graph whose vertices can be divided into two disjoint sets $U$ and $V$ such that every edge connects a vertex in $U$ to one in $V$, i.e., $U$ and $V$ are each independent sets. A complete bipartite graph $K_{m, n}$ is a special kind of bipartite graph where every vertex of the first vertex set is adjacent to every vertex of the second vertex set, where $m$ and $n$ are number of vertices in the first and second vertex set respectively. Let $G$ and $H$ be two graphs. The cartesian product or product $G \mathrm{WH}$ of $G$ and $H$ is a graph whose vertex set is the Cartesian product $V(G) \times V(H)$ and any two vertices $\left(u, u^{\prime}\right)$ and $\left(v, v^{\prime}\right)$ are adjacent in $G \mathrm{WH}$ if and only if either

- $u=v$ and $u^{\prime}$ is adjacent with $v^{\prime}$ in $H$, or

- $u^{\prime}=v^{\prime}$ and $u$ is adjacent with $v$ in $G$.

For a graph $G=(V, E)$ if $u, v \in V(G)$, then the distance $d(u, v)$ between $u$ and $v$ is defined as the length of a shortest $u-v$ path in $G$. The Wiener index of $G$ 
Pravin Garg and Shanu Goyal

is defined as

$$
W(G)=\sum_{\{u, v\} \subset V} d(u, v) .
$$

It is the oldest topological index and its mathematical properties and chemical applications have been extensively studied. The Wiener index was introduced by the chemist Harold Wiener in 1947 for explaining the correlation between the boiling points of paraffins and the structure of their molecules. It is a graph invariant much studied in both mathematical and chemical literature (see [12-14,17,21]). The concept of graph operator has found various applications in chemical research (see [8-11,16,18,19,22]).

The total graph $T(G)$ of a graph $G=(V, E)$ is that graph whose vertex set is $V \cup E$, and two vertices are adjacent if and only if they are adjacent or incident in $G$. It is introduced by Behzad \& Chartrand [5]. Several properties of total graphs are investigated in the literature (see $[1-4,6,7,15,20])$. The total graph $H=T(G)$ of $G$ is shown in Figure 1.

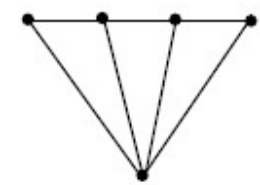

$\mathrm{F}_{1,4}$

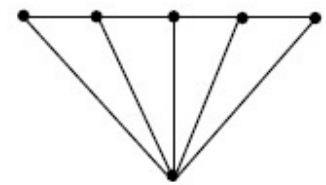

$\mathrm{F}_{1,5}$

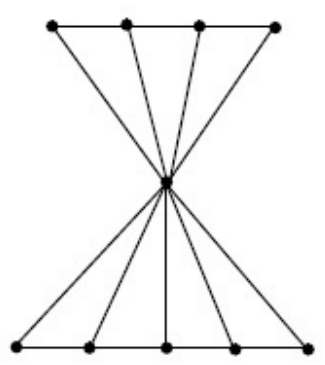

$\mathrm{F}_{1,4} \cdot \mathrm{F}_{1,5}$

Figure 1: A graph $G$ and its total graph $H=T(G)$

We define a new graph operator $F_{1, n} . F_{1, m}$ defined as follows: For a fan graph $F_{1, n}$, the graph $F_{1, n} . F_{1, m}$ is obtained by identifying a vertex corresponding to $\bar{K}_{1}$ of $F_{1, n}$ to one vertex corresponding to $\bar{K}_{1}$ of $F_{1, m}$. The graphs $F_{1,4}, F_{1,5}$ and $F_{1,4} \cdot F_{1,5}$ are shown in Figure 2.

\section{Wiener index of total graph of fan graphs}

Theorem 2.1. The Wiener index of the graph $G=F_{1, n}$,

$$
W(G)=n^{2}-n+1 .
$$

Proof: We know that

$$
W\left(F_{1, n}\right)=\sum_{u, v \in V\left(F_{1, n}\right)} d(u, v) .
$$

Therefore,

$$
\begin{aligned}
& =n \cdot 1+(n-1) \cdot 1+[(n-2)+(n-3)+\ldots+1] \cdot 2 \\
& =n^{2}-n+1
\end{aligned}
$$


On The Wiener Index of Some Total Graphs

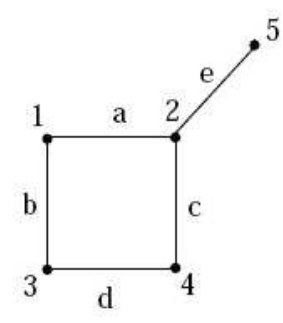

G

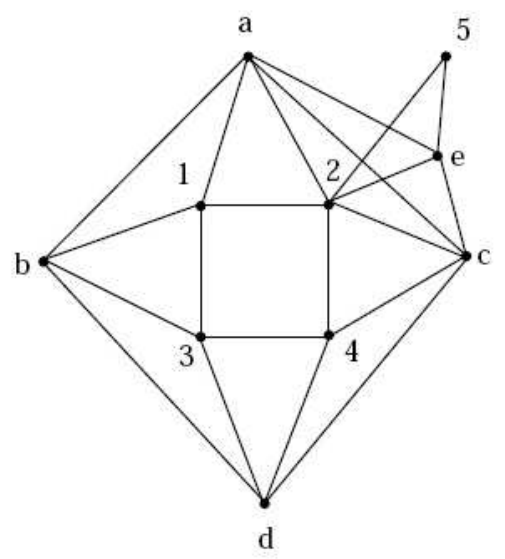

$\mathrm{H}$

Figure 2: The graphs $F_{1,4}, F_{1,5}, F_{1,4} \cdot F_{1,5}$

Theorem 2.2. The Wiener index of graph $G=T\left(F_{1, n}\right)$,

Proof: We know that

$$
W(G)=10 n^{2}-20 n+19 .
$$

$$
\begin{aligned}
& W\left(T\left(F_{1, n}\right)\right)=\sum_{u, v \in V\left(T\left(F_{1, n}\right)\right)} d(u, v) \\
& =\sum_{u, v \in V\left(F_{1, n}\right)} d(u, v)+\sum_{u \in V\left(F_{1, n}\right)} d(u, e)+\sum_{e \in E\left(F_{1, n}\right)} d(e, f) \\
& =W\left(F_{1, n}\right)+\sum_{u \in V\left(F_{1, n}\right)} d(u, e)+\sum_{e \in E\left(F_{1, n}\right)} d(e, f) .
\end{aligned}
$$

Here,

$$
\begin{aligned}
& W\left(F_{1, n}\right)=n^{2}-n+1 \quad \text { (usingTheo rem2.1) } \\
& \sum_{\substack{u \in V\left(F_{1, n}\right) \\
e \in E\left(F_{1, n}\right)}} d(u, e)=5 n^{2}-7 n+6
\end{aligned}
$$

and

$$
\sum_{e, f \in E\left(F_{1, n}\right)} d(e, f)=4 n^{2}-12 n+12 .
$$

Thus, 
Pravin Garg and Shanu Goyal

$$
\begin{aligned}
& W\left(T\left(F_{1, n}\right)\right)=\left(n^{2}-n+1\right)+\left(5 n^{2}-7 n+6\right)+\left(4 n^{2}-12 n+12\right) \\
& =10 n^{2}-20 n+19 .
\end{aligned}
$$

Hence,

$$
W\left(T\left(F_{1, n}\right)\right)=10 n^{2}-20 n+19 .
$$

Theorem 2.3. The Wiener index of graph $G=F_{2, n}$,

$$
W(G)=n^{2}+3 \text {. }
$$

Proof: We know that

Hence,

$$
\begin{aligned}
& W\left(F_{2, n}\right)=\sum_{u, v \in V\left(F_{2, n}\right)} d(u, v) \\
& =n .1+n .1+(n-1) \cdot 1+2+[(n-2)+(n-3)+\ldots . .+2+1] .2 \\
& =n^{2}+3 .
\end{aligned}
$$

$$
W\left(F_{2, n}\right)=n^{2}+3 \text {. }
$$

Theorem 2.4. The Wiener index of graph $G=T\left(F_{2, n}\right)$,

$$
W(G)=\frac{33 n^{2}-37 n+42}{2} .
$$

Proof: We know that

$$
\begin{aligned}
& W\left(T\left(F_{2, n}\right)=\sum_{u, v \in V\left(T\left(F_{2, n}\right)\right)} d(u, v)\right. \\
& =\sum_{u, v \in V\left(F_{2, n}\right)} d(u, v)+\sum_{u \in V\left(F_{2, n}\right)} d(u, e)+\sum_{e \in E\left(F_{2, n}\right)} d(e, f) \\
& =W\left(F_{2, n}\right)+\sum_{u \in V\left(F_{2, n}\right)} d(u, e)+\sum_{e \in E\left(F_{2, n}\right)} d(e, f) .
\end{aligned}
$$

Here,

$$
\begin{gathered}
W\left(F_{2, n}\right)=n^{2}+3 \quad \text { (usingTheorem2.3) } \\
\sum_{u \in V\left(F_{2, n}\right)} d(u, e)=7 n^{2}-n+4 \\
e \in E\left(F_{2, n}\right)
\end{gathered}
$$

and

$$
\sum_{e, f \in E\left(F_{2, n}\right)} d(e, f)=\frac{17 n^{2}-35 n+28}{2} .
$$

Thus,

$$
W\left(T\left(F_{2, n}\right)\right)=\left(n^{2}+3\right)+\left(7 n^{2}-n+4\right)+\frac{17 n^{2}-35 n+28}{2}
$$


On The Wiener Index of Some Total Graphs

$$
=\frac{33 n^{2}-37 n+42}{2} \text {. }
$$

Hence,

$$
W\left(T\left(F_{2, n}\right)=\frac{33 n^{2}-137 n+42}{2} .\right.
$$

Theorem 2.5. The Wiener index of graph $G=F_{1, m} . F_{1, n}$,

$$
W(G)=(m+n)^{2}-(m+n)+2 .
$$

Proof: We know that

$$
\begin{gathered}
W\left(F_{1, m} \cdot F_{1, n}\right)=\sum_{u, v \in V\left(F_{1, m} \cdot F_{1, n}\right)} d(u, v) \\
=m \cdot 1+n \cdot 1+(m-1) \cdot 1+[(m-2)+(m-3)+\ldots+1] \cdot 2+m n \cdot 2+(n-1) \cdot 1 \\
+[(n-2)+(n-3)+\ldots . .+1] \cdot 2 \\
=(m+n)^{2}-(m+n)+2 .
\end{gathered}
$$

Hence,

$$
W\left(F_{1, m} \cdot F_{1, n}\right)=(m+n)^{2}-(m+n)+2 .
$$

Theorem 2.6. The Wiener index of graph $G=T\left(F_{1, m} \cdot F_{1, n}\right)$,

$$
W(G)=10(m+n)^{2}-28(m+n)+41 .
$$

Proof: We know that

$$
\begin{gathered}
W\left(T\left(F_{1, m} . F_{1, n}\right)\right)=\sum_{u, v \in V\left(T\left(F_{1, m} \cdot F_{1, n}\right)\right)} d(u, v) \\
=\sum_{u, v \in V\left(F_{1, m} \cdot F_{1, n}\right)} d(u, v)+\sum_{u \in V\left(F_{1, m} \cdot F_{1, n}\right)} d(u, e)+\sum_{e \in E\left(F_{1, m} \cdot F_{1, n}\right)} d(e, f) \\
=W\left(F_{1, m} \cdot F_{1, n}\right)+\sum_{u \in V\left(F_{1, m} \cdot F_{1, n}\right)} d(u, e)+\sum_{e \in E\left(F_{1, m} \cdot F_{1, n}\right)} d(e, f) .
\end{gathered}
$$

Here,

$$
\begin{aligned}
& W\left(F_{1, m} \cdot F_{1, n}\right)=(m+n)^{2}-(m+n)+2 \quad \text { (usingTheorem2.5) } \\
& \sum_{u \in V\left(F_{1, m} \cdot F_{1, n}\right)} d(u, e)=5(m+n)^{2}-10(m+n)+12
\end{aligned}
$$

and

$$
\sum_{e, f \in E\left(F_{1, m} \cdot F_{1, n}\right)} d(e, f)=4(m+n)^{2}-17(m+n)+27 .
$$

Thus, 
Pravin Garg and Shanu Goyal

$$
\begin{aligned}
W\left(T\left(F_{1, m} . F_{1, n}\right)\right) & =\left((m+n)^{2}-(m+n)+2\right)+\left(5(m+n)^{2}-10(m+n)+12\right) \\
& \left.+4(m+n)^{2}-17(m+n)+27\right) \\
& =10(m+n)^{2}-28(m+n)+41 .
\end{aligned}
$$

Hence,

$$
W\left(T\left(F_{1, m} \cdot F_{1, n}\right)=10(m+n)^{2}-28(m+n)+41 .\right.
$$

\section{Wiener index of total graph of $K_{2} \mathrm{~W} C_{n}$}

Theorem 3.1. The Wiener index of the graph $G=K_{2} \mathrm{~W} C_{n}$,

Proof: Since,

$$
W(G)=\left\{\begin{array}{l}
\frac{n\left(n^{2}+2 n-1\right)}{2}, \text { nisodd } \\
\frac{n^{2}(n+2)}{2}, \text { niseven } .
\end{array}\right.
$$

$$
W\left(K_{2} \mathrm{~W} C_{n}\right)=\sum_{u, v \in V\left(2 C_{n}\right)} d(u, v)
$$

To calculate $W\left(K_{2} \mathrm{~W} C_{n}\right)$, we consider two cases:

Case 1: Suppose $n$ is odd. Then,

Thus,

$$
W\left(K_{2} \mathrm{~W}_{n}\right)=3 n .1+4 n\left[2+3+\ldots . .+\frac{n-1}{2}\right]+2 n \cdot\left(\frac{n-1}{2}+1\right) .
$$

$$
W\left(K_{2} \mathrm{~W} C_{n}\right)=\frac{n\left(n^{2}+2 n-1\right)}{2} .
$$

Case 2: Suppose $n$ is even. Then,

$$
W\left(K_{2} \mathrm{~W}_{n}\right)=3 n \cdot 1+4 n\left[2+3+\ldots \ldots+\left(\frac{n}{2}-1\right)\right]+3 n \cdot \frac{n}{2}+n \cdot\left(\frac{n}{2}+1\right)
$$

Thus,

$$
W\left(K_{2} \mathrm{~W} C_{n}\right)=\frac{n^{2}(n+2)}{2}
$$

Theorem 3.2. The Wiener index of graph $G=T\left(K_{2} \mathrm{~W} C_{n}\right)$,

$$
W\left(T\left(K_{2} \mathrm{~W} C_{n}\right)\right)=3 n^{3}+9 n^{2}+2 .
$$

Proof: We know that

$$
\begin{aligned}
& W\left(T\left(K_{2} \mathrm{~W} C_{n}\right)\right)=\sum_{u, v \in V\left(T\left(K_{2} \mathrm{~W}_{n}\right)\right)} d(u, v) \\
& =\sum_{u, v \in V\left(K_{2} \mathrm{~W} C_{n}\right)} d(u, v)+\sum_{u \in V\left(K_{2} \mathrm{~W} C_{n}\right)} d(u, e)+\sum_{e \in E\left(K_{2} \mathrm{~W} C_{n}\right)} d(e, f)
\end{aligned}
$$


On The Wiener Index of Some Total Graphs

$$
=W\left(K_{2} \mathrm{~W} C_{n}\right)+\sum_{u \in V\left(K_{2} \mathrm{~W} C_{n}\right)} d(u, e)+\sum_{e \in E\left(K_{2} \mathrm{~W} C_{n}\right)} d(e, f)
$$

To calculate $W\left(T\left(K_{2} \mathrm{~W} C_{n}\right)\right)$, we consider two cases:

Case 1: Suppose $n$ is odd. Then,

Therefore,

$$
\begin{gathered}
W\left(K_{2} \mathrm{~W} C_{n}\right)=\frac{n\left(n^{2}+2 n-1\right)}{2} \quad \text { (usingTheorem3.1) } \\
\sum_{u \in V\left(K_{2} \mathrm{~W} C_{n}\right)} d(u, e)=\frac{n\left(3 n^{2}+12 n+1\right)}{2} \\
\sum_{e \in E\left(K_{2} \mathrm{~W} C_{n}\right)} d(e, f)=n^{3}+2 n^{2}+n .
\end{gathered}
$$

$$
\begin{aligned}
& W\left(T\left(K_{2} \mathrm{~W} C_{n}\right)\right)=\frac{n\left(n^{2}+2 n-1\right)}{2}+\frac{n\left(3 n^{2}+12 n+1\right)}{2}+\left(n^{3}+2 n^{2}+n\right) \\
& =3 n^{3}+9 n^{2}+n .
\end{aligned}
$$

Case 2: Suppose $n$ is even. Then,

$$
\begin{gathered}
W\left(K_{2} \mathrm{~W} C_{n}\right)=\frac{n^{2}(n+2)}{2} \quad \text { (usingTheorem3.1) } \\
\sum_{u \in V\left(K_{2} \mathrm{~W} C_{n}\right)} d(u, e)=\frac{3 n^{2}(n+4)}{2} \\
e \in E\left(K_{2} \mathrm{~W} C_{n}\right)
\end{gathered}
$$

and

$$
\sum_{e, f \in E\left(K_{2} \mathrm{WC}_{n}\right)} d(e, f)=n^{3}+2 n^{2}+n .
$$

Therefore,

$$
\begin{aligned}
& W\left(T\left(K_{2} \mathrm{~W} C_{n}\right)\right)=\frac{n^{2}(n+2)}{2}+\frac{3 n^{2}(n+4)}{2}+\left(n^{3}+2 n^{2}+n\right) \\
& =3 n^{3}+9 n^{2}+n
\end{aligned}
$$

Hence,

$$
W\left(T\left(K_{2} \mathrm{~W} C_{n}\right)\right)=3 n^{3}+9 n^{2}+n
$$

\section{Wiener index of total graph of $K_{m, n}$}

Theorem 4.1. The Wiener index of the graph $G=T\left(K_{m, n}\right)$,

$$
W(G)=\frac{1}{2}\left[\left(2 m^{2}+2\right)\left(n^{2}+1\right)+(3 m n-2)(m+n)-2(m n+1)\right]
$$


Pravin Garg and Shanu Goyal

Proof: Since,

$$
\begin{aligned}
& W\left(T\left(K_{m, n}\right)\right)=\sum_{u, v \in V\left(T\left(K_{m, n}\right)\right)} d(u, v) \\
& =\sum_{u, v \in V\left(K_{m, n}\right)} d(u, v)+\sum_{e, f \in E\left(K_{m, n}\right)} d(e, f)+\sum_{u \in V\left(K_{m, n}\right),} d(u, e) .
\end{aligned}
$$

Now,

$$
\begin{gathered}
\sum_{u, v \in V\left(K_{m, n}\right)} d(u, v)=m n(1)+\left({ }^{m+n} C_{2}-m n\right)(2) \\
\sum_{u \in V\left(K_{m, n}\right),} d(u, e)=2 m n(1)+(m(m n-n)+n(m n-m))(2) \\
\sum_{e \in E\left(K_{m, n}\right)} d(e, f)=\frac{m^{2} n+n^{2} m-2 m n}{2}(1)+\frac{m^{2} n^{2}-m^{2} n-n^{2} m+m n}{2}(2) .
\end{gathered}
$$

Thus,

$$
\begin{aligned}
W\left(T\left(K_{m, n}\right)\right)= & \left(m n(1)+\left({ }^{m+n} C_{2}-m n\right)(2)\right)+(2 m n(1)+(m(m n-n)+n(m n-m))(2)) \\
& +\left(\frac{m^{2} n+n^{2} m-2 m n}{2}(1)+\frac{m^{2} n^{2}-m^{2} n-n^{2} m+m n}{2}(2)\right) \\
& =\frac{1}{2}\left[\left(2 m^{2}+2\right)\left(n^{2}+1\right)+(3 m n-2)(m+n)-2(m n+1)\right]
\end{aligned}
$$

\section{Conclusion}

In this article, we have investigated the results related to the Wiener index of the total graph of five particular graphs, namely, $F_{1, n}, F_{2, n}, F_{1, n} . F_{1, m}, K_{2} \mathrm{~W} C_{n}$ and $K_{m, n}$.

Acknowledgement. Research is supported by the University Grants Commission, New Delhi, India under the UGC-BSR Project No.F.30-84/2014(BSR).

\section{REFERENCES}

1. M.M.Akbar Ali and S.Panayappan, Cycle multiplicity of total graph of $C_{n}, P_{n}$ and $K_{1, n}$, International Journal of Engineering, Science and Technology, 2(2) (2010) 54-58.

2. M.Behzad, A criterion for the planarity of the total graph of a graph, Math. Proc. Cambridge Philos. Soc., 63 (1967) 679-681.

3. M.Behzad, The connectivity of total graphs, Bull. Aust. Math. Soc., 1 (1969) 175-181.

4. M.Behzad, A characterization of total graphs, Proc. Amer. Math. Soc., 26(3) (1970) 383-389.

5. M.Behzad and G.Chartrand, Total graphs and traversability, Proc. Edinb. Math. Soc., 15(2) (1966) 117-120.

6. M.Behzad and H.Radjavi, The total group of a graph, Proc. Amer. Math. Soc., 19 (1968) 158-163.

7. M.Behzad and H.Radjavi, Structure of regular total graphs, J. Lond. Math. Soc., 44 
(1969) 433-436.

8. A.A.Dobrynin and L.S.Mel'nikov, Wiener index for graphs and their line graphs, Diskretn. Anal. Issled. Oper. Ser. 2, 11(2004), 25-44, in Russian.

9. A.A.Dobrynin and L.S.Mel'nikov, Trees, quadratic line graphs and the Wiener index, Croat. Chem Acta, 77 (2004) 477-480.

10. A.A.Dobrynin and L.S.Mel'nikov, Wiener index for graphs and their line graphs with arbitrary large cyclomatic numbers, Appl. Math. Lett., 18 (2005) 307-312.

11. A.A.Dobrynin and L.S.Mel'nikov, Wiener index, line graphs and the cyclomatic number, MATCH Commun. Math. Comput. Chem., 53 (2005) 209-214.

12. A.A.Dobrynin, R.Entringer and I.Gutman, Wiener index of trees: Theory and applications, Acta Appl. Math., 66 (2001) 211-249.

13. A.A.Dobrynin, I.Gutman, S.Klav $\breve{z}$ ar and P. $\breve{Z}$ igert, Wiener index of hexagonal systems, Acta Appl. Math., 72 (2002) 247-294.

14. R.C.Entringer, Distance in graphs: Trees, J. Combin. Math. Combin. Comput., 24 (1997) 65-84.

15. F.Gavril, A recognition algorithm for the total graphs, Networks, 8(2) (1978) 121-133.

16. I.Gutman and E.Estrada, Topological indices based on the line graph of the molecular graph, J. Chem. Inf. Comput. Sci., 36 (1996) 541-543.

17. I.Gutman, Y.N.Yeh, S.L.Lee and Y.L.Luo, Some recent results in the theory of the Wiener number, Indian J. Chem., 32A (1993) 651-661.

18. I.Gutman, L.Popovic, B.K.Mishra, M.Kaunar, E.Estrada and N.Guevara, Application of line graphs in physical chemistry. Predicting surface tension of alkanes, J. Serb. Chem. Soc., 62 (1997) 1025-1029.

19. K.Das, Algorithms to find wiener index of some graphs, Annals of Pure and Applied Mathematics, 7(1) (2014) 13-18.

20. S.Kavitha and S.Lavanya, Fuzzy chromatic number of line, total and middle graphs of fuzzy complete graphs, Annals of Pure and Applied Mathematics, 8(2) (2014) 251-260.

21. M.H.Khalifeh, H.Yousefi-Azari, A.R.Ashrafi and S.G.Wagner, Some new results on distance-based graph invariants, European J. Combin., 30(5) (2009) 1149-1163.

22. K.Thilakam and A.Sumathi, Wiener index of a cycle in the context of some graph operations, Annals of Pure and Applied Mathematics, 5(2) (2014) 183-191. 